EPJ Web of Conferences 71, 00090 (2014)

DOI: 10.1051/epjconf/20147100090

(C) Owned by the authors, published by EDP Sciences, 2014

\title{
Oscillatory modes of quarks in baryons for 3 quark flavors $u, d, s$
}

\section{Tuning to harmonic numbers of oscimodes of baryons}

\author{
Peter Minkowski ${ }^{1,3, a}$ and Sonia Kabana ${ }^{2, b}$ \\ ${ }^{1}$ Albert Einstein Center for Fundamental Physics - ITP, University of Bern, Switzerland. \\ ${ }^{2}$ SUBATECH, Ecole des Mines, 4 rue Alfred Kastler, 44307 Nantes, France. \\ ${ }^{3} \mathrm{PH}-\mathrm{TH}$ Division CERN.
}

\begin{abstract}
The present notes prepare the counting of 'oscillatory modes of $N_{f l}=3$ light quarks', $-\mathrm{u}, \mathrm{d}, \mathrm{s}-$, using the $\mathrm{SU}\left(2 N_{f l}=6\right) \times \operatorname{SO} 3(\vec{L})$

broken symmetry classification, extended to the harmonic oscillator symmetry of 3 paired oscillator modes . $\vec{L}=\sum_{n=1}^{N} \vec{L}_{n}$ stands for the space rotation group generated by the sum of the 3 individual angular momenta of quarks in their c.m. system . The motivation arises from modeling the yields of hadrons in heavy ion collisions at RHIC and LHC, necessitating at the respective highest c.m. energies per nucleon pairs an increase of heavy hadron resonances relative to e.g. SPS energies, whence the included hadrons are treated as a noninteracting gas .
\end{abstract}

\section{Preamble}

Perspectives : A. How to count oscillatory modes of quarks in baryons for 3 quark flavors $\mathrm{u}, \mathrm{d}, \mathrm{s}$ $-t \geq 0$

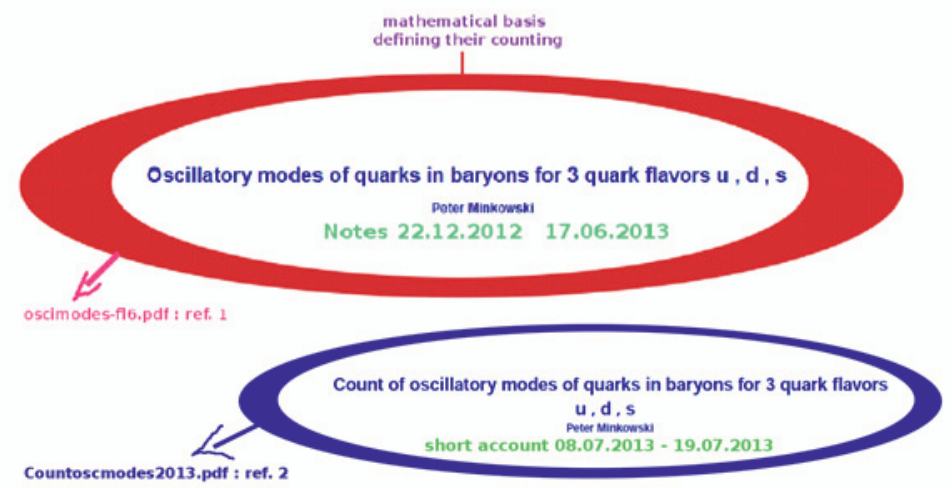

Fig. 1 : ref. $1=[1] ;$ ref. $2=[2]$.

\footnotetext{
a e-mail: mink@itp.unibe.ch

be-mail: SoniaKabana<kabana@mail.cern.ch>
}

This is an Open Access article distributed under the terms of the Creative Commons Attribution License 2.0, which permits unrestricted use, distribution, and reproduction in any medium, provided the original work is properly cited. 
B. Tuning to harmonic numbers of oscimodes of baryons

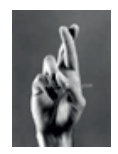

Fig. 2 : Some definite results are presented in section 5.

\section{Introduction}

The perspectives illustrated in Figs. 1 and 2 are meant to apply to present and future derivations . In this sense refs. 1 and $2-[1]$ and [2] - refer to recent results. The comparison of hadron yields measured at RHIC and LHC with a noninteracting hadron resonance gas necessitates the counting of these resonances, which is not obvious. This is illustrated in Fig. 1 of ref. 3 [3], reproduced as Fig. 3 below. Thus the problem of identifying 'oscillatory modes of light quark flavors in baryons', presented in ref. $4-[4]$ - took center-stage. ${ }^{1}$ Having read in 1976 a paper on relativistic oscillator solutions to the Dirac equation, only recently I found its source, which becomes ref. $18-[18]{ }^{2}$

Here we focus on the cornerstones, which allow to count these oscillatory modes, as outlined in extenso in refs. 1 and 2 , op.cit. , beginning with the classification of the representations of $S_{3}$ - the permutation group of the three quarks in configuration space - arising through the induced representation from the associated wave functions, in the subsequent sections.

\section{Factoring out the approximate symmetry group in spin-flavor space as well as overall color}

It is inherent to the path followed to point out the historical formulation of the 'bootstrap hypothesis' underlying and restricting the full set of S-matrix elements pertaining to strongly interacting hadrons, due to G. F. Chew e.g. in ref. 5 - [5]. A good textbook reference is ref. 6 - [6].

In this context all resonances observed or hypothetically to be observed in scattering of stable hadrons, irrespective of their width, are considered to be hadrons. A central notion within the 'bootstrap'framework is the density of hadrons and its limiting behaviour for large mass-square

3

$$
\varrho_{n}\left(m^{2}\right)=\frac{\partial \mathbb{N}\left(m^{2}\right)}{\partial m^{2}}
$$

In the density with respect to mass-square $\varrho_{n}$ defined in eq. 1 the density per phase space of an isolated state of momentum $\vec{p}$ is not included

$$
d \Phi=V(2 \pi)^{-3} d^{3} p ; V: \text { space volume }
$$

1 What comes to my mind in this context as a negative example is the 'Limerick'-song by Georg Kreisler : "Wenn ich Inspiration such les ich im Telefonbuch und zwar auf Seite V ." $\longleftrightarrow$ 'When I search for inspiration I read the phone book and that on page V' . (free translation)

2 I am indebted to Christoph Greub for reminding me of the first article by C. L. Critchfield - [18] - on scalar potentials .

3 We use the symbol $\mathbb{N}$ for numbering integers except the number of valence quarks forming a baryon, denoted $N$. 


\section{ICNFP 2013}

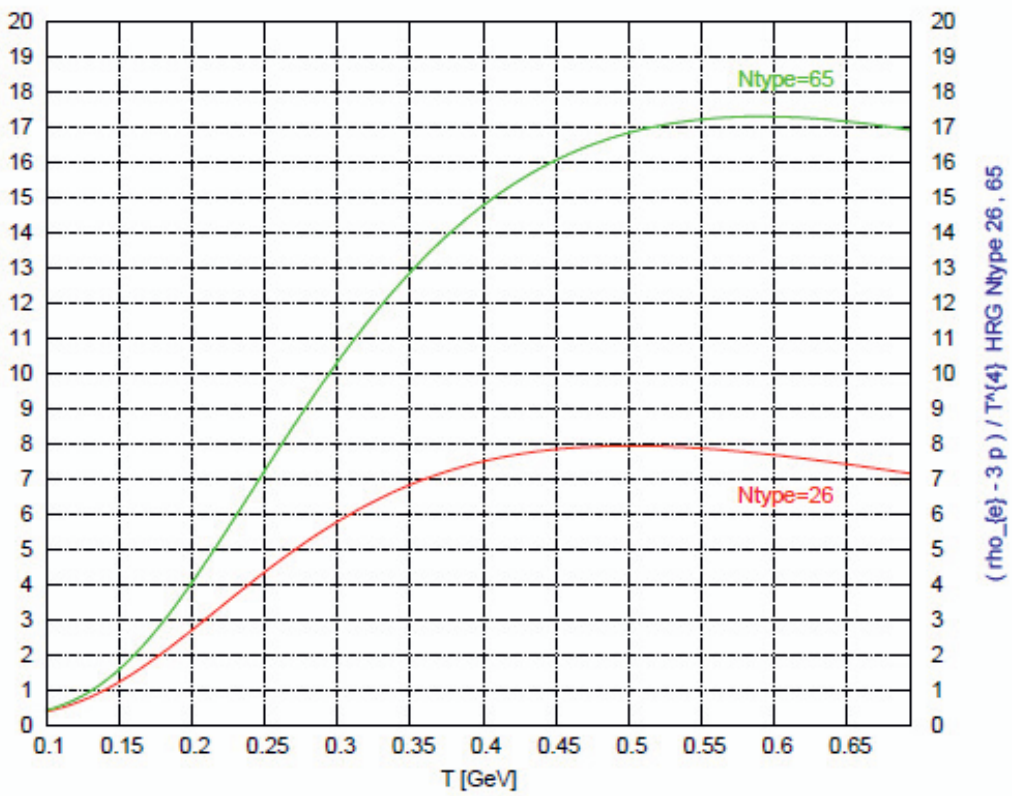

Fig. 3 : The thermal quantity representing the trace anomaly - dscale $(T)=\left(\varrho_{e}-3 p\right) / T^{4}$ is shown for $0.1 \mathrm{GeV} \leq T \leq 0.7 \mathrm{GeV}$. The comparison of HRG collections Ntype=65 and 26 shows the sensitive temperature regions beyond temperatures where chemical freeze out takes place $-T \sim 150-170 \mathrm{MeV}$ for $\mathrm{Pb}-\mathrm{Pb}$ collisions at SPS and $\mathrm{Au}-\mathrm{Au}$ collisions at RHIC.

The parametrization defined in eq. 1 is directly applicable to counting resonances in the particle listings of the PDG [7]. To this end a binning in mass-square is to be chosen and a histogram of resonance counts per bin yields the so approximated density function $\varrho_{n}$.

In 1965 Rolf Hagedorn ( 1919 - 2003 ) wrote an elaborate paper - [8] - centered around the hypothesis of the limiting behaviour of the quantity $\varrho_{n}$ for $m \rightarrow \infty$ as a solution to the bootstrap conditions

$$
\begin{aligned}
& \varrho_{n}\left(m^{2}\right) \sim\left(\frac{m^{2}}{m_{0}^{2}}\right)^{a} \exp \left(m / T_{0}\right) \text { for } m \rightarrow \infty \\
& m_{0}, a, T_{0}: \text { characteristic parameters }
\end{aligned}
$$

In 1968 Gabriele Veneziano - [9] - arrived at a solution to the 'bootsrap' idea starting from the decay amplitude for the process

$$
\omega \rightarrow \pi^{+} \pi^{-} \pi^{0}
$$

reproducing Regge poles in all two particle channels upon suitable extrapolations in their respective momenta, with generalizations to multiparticle ampltudes called 'dual'. The structure underlying the totality of dual amplitudes is the quantum mechanical motion of a one dimensional open (super)string with constant string tension $T=1 /\left(2 \pi \alpha^{\prime}\right)$, whose harmonic vibrations generate linear $\left(J\right.$ versus $\left.\alpha^{\prime} \mathrm{m}^{2}\right)$ bosonic and fermionic Regge trajectories. A review may be found in ref. 10=[10]. In the domain of numbers the counting of partitions, i.e. the power of the set of nonnegative integers $n_{1}, n_{2}, \cdots, n_{\infty}$ with $n_{k}=0,1, \cdots, \infty \forall k$

$$
\begin{aligned}
& \wp(\mathbb{N})=\left\{n_{1}, n_{2} \cdots n_{\infty} \mid \sum_{k=1}^{\infty} k n_{k}=\mathbb{N}\right\} \\
& n_{1}, n_{2} \cdots n_{\infty}=0,1, \cdots
\end{aligned}
$$


determines by its asymptotic behaviour for $\mathbb{N} \rightarrow \infty$ that indeed the free superstring possesses a similar growth as in eq. 3 - modulo multiplicative logarithmic factors in the exponent - such that a maximal temperature exists in accordance with Hagedorn's hypothesis . This is worked out in ref. 11 - [11] . In a specific case of open superstrings on noncommutative space eq. 3 is reproduced with $a=-\frac{9}{4}$ in ref. $12-[12] . \wp(\mathbb{N})$ in eq. 5 is treated in ref. $13-[13]$, pp. $822 \mathrm{ff}$.

\subsection{QCD as local field theory in $d=3+1$ uncurved dimensions as antithesis to limiting temperature}

For clarity of thought with respect to internal consistency of dual amplitudes, as originally proposed in 1968 by Gabriele Veneziano - [9] - it must be emphasized that this was achieved through several stages within about 16 years as illustrated e.g. in ref. $14-[14]$ - and continues to be vigorously investigated. The emerging theory of superstrings indeed generates oscillatory modes of open and closed superstring vibrations, including gravitons - easiest in 10 or 11 space-time dimensions - and thus a quantized version of gravity. On the side of symmetries the said consistency requirements imply manifest supersymmetries, which can be exactly conserved in part or completely broken .

Let us turn towards the title of this subsection and counterpose the hypothesis that there exists an essentially unique consistent local field theory in the limiting case of uncurved $d=3+1$ space time - called QCD today - with the following properties :

a) describes strong hadronic interactions

b) admits perturbative as well as nonperturbative renormalizability

in the ultraviolet limit as appropriate for uncurved space-time

c) accounts for the composite nature of hadrons as composed of at least several flavors of $q \bar{q}^{\prime}$ fields for ordinary mesons and $q q^{\prime} q^{\prime \prime}\left(\bar{q} \bar{q}^{\prime} \bar{q}^{\prime \prime}\right)$ fields for associated baryons ( anti-baryons ) respectively

As a textbook illuminating also aspects of thermodynamics in local field theories the book by Jean Zinn-Justin - ref. 15 : [15] - shall be quoted. The requirement or postulate of uniqueness as invoked by Geoffrey Chew needs at least a reformulation in view of the enlarged framework of dual amplitudes. Here we shall discuss a particular consequence of the premises a) - c) above - to construct oscillatory modes of a finite number of field theoretic degrees of freedom - next .

\subsection{Poincaré-invariant harmonic oscillator modes for baryons in the configuration space of barycentric coordinates for 3 valence quarks $u, d, s$}

Seen from the time line of the first years of the 70-th it took about 9 years until an explicit construction of oscillator modes of baryons exhibiting a density function

$$
\begin{aligned}
& \varrho_{n}\left(m^{2}\right) \sim\left(\frac{m^{2}}{m_{0}^{2}}\right)^{u} \text { for } m \rightarrow \infty \\
& m_{0}, u: \text { characteristic parameters and } u=\text { positive integer }
\end{aligned}
$$

for valence quark (antiquark) configurations in mesons and baryons - [4] - compatible with a finite number of degrees of freedom.

We envisage baryons within QCD, allowing temporarily the local color gauge group to be generalized from 3 colors to $1<N_{c} \rightarrow N$ to simplify notation, whenever no confusion arises. Baryons are: 
fermions for $N$ odd, bosons for $N$ even. A concise sketch of barycentric canonically conjugate momentum and spatial coordinates - pairs of three vectors

$$
\left(\vec{\pi}_{v}, \vec{z}_{v}\right) ; v=1,2, \cdots, N
$$

follows.

\section{barycentric coordinates in the overall c.m. system}

$$
\begin{aligned}
& \vec{\pi}_{1}=\frac{1}{\sqrt{2}}\left(\vec{p}_{1}-\vec{p}_{2}\right) \quad, \quad \vec{z}_{1}=\frac{1}{\sqrt{2}}\left(\vec{x}_{1}-\vec{x}_{2}\right) \\
& \vec{\pi}_{2}=\frac{1}{\sqrt{6}}\left(\vec{p}_{1}+\vec{p}_{2}-2 \vec{p}_{3}\right), \vec{z}_{2}=\frac{1}{\sqrt{6}}\left(\vec{x}_{1}+\vec{x}_{2}-2 \vec{x}_{3}\right) \\
& \vec{\pi}_{v}=(v(v+1))^{-1 / 2}\left(\begin{array}{cc}
\sum_{\alpha=1}^{v} & \vec{p}_{\alpha} \\
-v & \vec{p}_{v+1}
\end{array}\right), \vec{z}_{v}=(v(v+1))^{-1 / 2}\left(\begin{array}{cc}
\sum_{\alpha=1}^{v} & \vec{x}_{\alpha} \\
-v & \vec{x}_{v+1}
\end{array}\right) \\
& \vec{\pi}_{N-1}=\cdots \quad, \quad \vec{z}_{N-1}=\cdots \\
& \vec{\pi}_{N}=N^{-1 / 2} \sum_{\alpha=1}^{N} \vec{p}_{\alpha} \rightarrow 0, \vec{z}_{N}=N^{-1 / 2} \sum_{\alpha=1}^{N} \vec{x}_{\alpha} \rightarrow 0
\end{aligned}
$$

The last line in eq. 8 refers to c.m. momentum and position, both fixed to vanish they do not represent genuine degrees of freedom.

\section{Completing canonically conjugate variables with the pair $\mathcal{L}_{N}, t$}

In the local Lagrangean density setting of QCD the external quark masses, denoted $M_{q}$, appropriate multipliers of the scalar densities $\bar{q} q$ composing the mass term, enter in the form, also discussed recently in refs. $16-[16]-$ and $17-[17]-$

$$
-\mathcal{L}_{q-\text { mass }}=\sum_{\text {flavors }} \frac{z_{q}}{z_{M}} M_{q} \bar{q}^{c} q^{c}
$$

The quark masses $M_{q}$ are independent of global c.m. frame time $t$ and of configuration space variables $\vec{z}_{v} ; v=1,2, \cdots, N-1$; the mass functions $m_{q}$ do depend on the entire spatial configurarion of the (resonance-) state considered - always reduced to the overall c.m. system - and therein of time $t$.

For the dynamical meaning of c.m. time the implication of the last relation in eq. 8 repeated below

$$
\vec{\pi}_{N}=N^{-1 / 2} \sum_{\alpha=1}^{N} \vec{p}_{\alpha} \rightarrow 0 \quad, \quad \vec{z}_{N}=N^{-1 / 2} \sum_{\alpha=1}^{N} \vec{x}_{\alpha} \rightarrow 0
$$

is central : the 'prima facie' canonical pair $\vec{\pi}_{N}, \vec{z}_{N}$ in eq. 10 is removed from the set of dynamical canonical (3-vector-)variable pairs

$$
\left(\vec{\pi}_{1}, \vec{z}_{1}\right), \cdots,\left(\vec{\pi}_{N-1}, \vec{z}_{N-1}\right) ; \vec{\pi}_{N}=0, \vec{z}_{N}=0
$$

maintaining kinematic simultaneous vanishing displayed in eq. 11 .

Configuration space variables $\vec{x}_{v} ; v=1, \cdots N$ inherit from the relations displayed in eqs. 10 and 11 the following c.m.-equivalence

$$
\vec{x}_{v} \underset{c . m .}{\sim} \vec{x}_{v}-\vec{X} ; \quad v=1, \cdots, N ; \vec{X}=\frac{1}{N}\left(\sum_{\varrho=1}^{N} \vec{x}_{\varrho}\right)
$$


which implies that each $\vec{x}_{v}$ is equivalent to a homogeneous linear combination of the barycentric coordinates $\vec{z}_{\kappa} ; 1 \leq \kappa \leq N-1$. c.m. time $t$ is defined as common time coordinate of four vectors

$$
\begin{aligned}
& \vec{x}_{v} \rightarrow\left\{x^{\mu}\right\}_{v}=\left(t, \vec{x}_{v}\right) ; \quad \begin{array}{l}
\mu=0,1,2,3 \\
v=1, \cdots, N
\end{array} \\
& \text { consistent with the constraint : } \sum_{v=1}^{N} \vec{x}_{v}=0 ; \\
& \text { remembering } N=N_{c}
\end{aligned}
$$

\section{Orbits in configuration space and velocities}

The configuration space variables obeying c.m.-constraints and c.m.-equivalence are together with c.m.-time as displayed in eq. 13

$$
\left(t, \vec{x}_{1}\right),\left(t, \vec{x}_{2}\right), \cdots,\left(t, \vec{x}_{N}\right) ; \sum_{\varrho=1}^{N} \vec{x}_{\varrho}=0
$$

with $\vec{x}_{v} ; v=1, \cdots, N$ conceived independent of $\mathrm{t}$ - whereas an orbit is formed by binding the space-variables $\vec{x}_{v}=f_{v}(t) \equiv \vec{x}_{v}(t)$, as expressed in the next equation

$$
\begin{aligned}
& \left(t, \vec{x}_{1}\right) \quad,\left(t, \vec{x}_{2}\right) \quad, \cdots,\left(t, \vec{x}_{N}\right) \quad ; \sum_{\varrho=1}^{N} \vec{x}_{\varrho}=0 \\
& \left(t, \vec{x}_{1}(t)\right),\left(t, \vec{x}_{2}(t)\right), \cdots,\left(t, \vec{x}_{N}(t)\right) ; \sum_{\varrho=1}^{N} \vec{x}_{\varrho}(t)=0
\end{aligned}
$$

The definition of orbit in the second relation in eq. 15 leads to the definition of velocities maintaining c.m.-constraints and c.m.-equivalence

$$
\begin{aligned}
& \vec{v}_{v}(t)=\frac{d}{d t} \vec{x}_{v}(t) \equiv \dot{\vec{x}}_{v} ; v=1, \cdots, N ; \\
& \text { with : } \sum_{\varrho=1}^{N} \vec{v}_{\varrho}(t)=0
\end{aligned}
$$

\section{The main quantities $\mathcal{L}_{N}$ depends on}

These quantities are compiled in the list of 3 items below

1) a universal scale parameter of dimension mass-square $\Lambda=\left(2 \alpha^{\prime}\right)^{-1}$ related to the inverse slope of Regge trajectories, as well as quark masses, minimally $M_{q} ; q=u, d, s$, composing the mass term in the local QCD Lagrangean given in eq. 9, where the finite renormalization constants $z_{q}, z_{m}$ are to be chosen to implement exact Ward identities, which in turn specify $M_{q}-[16]$, [17].

2) quark mass functions $m_{\varrho}\left(M_{q} ;\left\{\vec{z}_{v}\right\}\right) ; v=1, \cdots, N-1 ; \varrho=1, \cdots, N$

3) velocities $\vec{v}_{v}(t) \equiv \dot{\vec{x}}_{v} ; v=1, \cdots, N$; with : $\sum_{\varrho=1}^{N} \vec{v}_{\varrho}(t)=0$

This leads to the Ansatz - [4] - for light and heavy quark flavors remembering that we use rational units with $\hbar=c=1$.

$$
\begin{aligned}
& \mathcal{L}_{N}=-\sum_{\alpha=1}^{N}\left[m_{\alpha}^{2}-\sum_{\beta=1}^{N} \sum_{\gamma=1}^{N} Q_{\beta \gamma}^{\alpha} \vec{v}_{\beta} \vec{v}_{\gamma}\right]^{1 / 2} \\
& m_{\alpha}=m_{\alpha}\left(M_{\delta}, \Lambda ; \underline{z}\right) ; Q_{\beta \gamma}^{\alpha}=Q_{\beta \gamma}^{\alpha}\left(M_{\delta}, \Lambda ; \underline{z}\right) \\
& \underline{z}=\left\{\vec{z}_{1}, \cdots, \vec{z}_{N-1}\right\}
\end{aligned}
$$


The limiting quantities in the chiral limit obtain as follows

$$
\begin{aligned}
& m_{\alpha}\left(M_{\delta}, \Lambda ; \underline{z}\right) \sim \stackrel{\circ}{m}_{\alpha}(\Lambda ; \underline{z})+M_{\alpha} \quad+o\left(M_{\delta}\right) \\
& Q_{\beta \gamma}^{\alpha}\left(M_{\delta}, \Lambda ; \underline{z}\right) \sim \stackrel{\circ}{Q}_{\beta \gamma}^{\alpha}(\Lambda ; \underline{z})+M_{\varrho} A_{\beta \gamma}^{\varrho \mid \alpha} \Lambda^{\frac{1}{2}}+o\left(M_{\delta} \Lambda^{\frac{1}{2}}\right) \\
& \text { for } M_{\delta} \rightarrow 0
\end{aligned}
$$

In ref. $4-[4]$ the quantities

$$
\begin{array}{ccc}
m_{\alpha}\left(M_{\delta}, \Lambda ; \underline{z}\right) & \underset{\stackrel{\circ}{m}_{\alpha}}{\longrightarrow}(\Lambda ; \underline{z}) \\
& \text { chiral limit } & \\
Q_{\beta \gamma}^{\alpha}\left(M_{\delta}, \Lambda ; z\right) & \longrightarrow & \stackrel{\circ}{Q}_{\beta \gamma}^{\alpha}(\Lambda ; \underline{z})
\end{array}
$$

defined in eq. 18 were identified but shall be kept distinct here, for clarity of logic .

\section{Remarks with respect to the chiral limit}

$\chi 1$ The discussion of oscillatory modes of baryons in the chiral limit is simplified but the phenomenological application is limited to the light flavors $\mathrm{u}, \mathrm{d}, \mathrm{s}$.

$\chi 2$ In the zero width approximation the appearance of 8 massless pseudoscalar goldstone bosons

$$
\begin{aligned}
& \stackrel{\circ}{\pi}_{\sigma}=\left(\pi^{+}, \pi^{0}, \pi^{-} ; \bar{K}^{0}, \bar{K}^{-} ; K^{+}, K^{0} ; \eta\right) ; \\
& \sigma=1, \cdots, 8 ; m_{\pi_{\sigma}}=0
\end{aligned}
$$

does not enter directly, but the modes with large masses will decay to lower ones and one ore more $\stackrel{\circ}{\pi}-\mathrm{s}-$ also in cascades. This is after removing infrared divergencies not significantly different for the realistic case of finite $\pi_{\sigma}$ - masses. As a consequence such cascades of excited baryon resonance decays generate a pronounced feeding problem, aggravated if heavy ion collisions are performed at todays energy frontiers at RHIC and LHC, less so at FAIR .

$\chi 3$ In addition at high energy also hevay flavors $\mathrm{c}, \mathrm{b}, \mathrm{t}$ are or will be copiously produced aggravating the study of light flavored (anti-)baryons .

On the phenomenological side the $\Delta$ Regge trajectory from ref. $19-[19]$ - is shown in Fig. 4 , in which the additional trajectories were erased .

\section{$\mathcal{L}_{N} \rightarrow \stackrel{\circ}{\mathcal{L}}_{N}$ in the chiral limit}

For logical consistency we can consider the chiral limit of arbitrary $N=N_{c}$ and $N_{f l}$ light flavors of quark, as long as $N>1$ and $N>\frac{2}{11} N_{f l}$, ensuring asymptotic ( ultraviolet) freedom .

The Ansatz for $\stackrel{\circ}{\mathcal{L}}_{N}$ becomes

$$
\stackrel{\circ}{Q}_{\beta \gamma}^{\alpha}=\stackrel{\circ}{m}_{\alpha}^{2} \frac{1}{K_{N}} \delta_{\beta \gamma} ; K_{N}: \text { dimensionless, positive constant }
$$




\section{EPJ Web of Conferences}

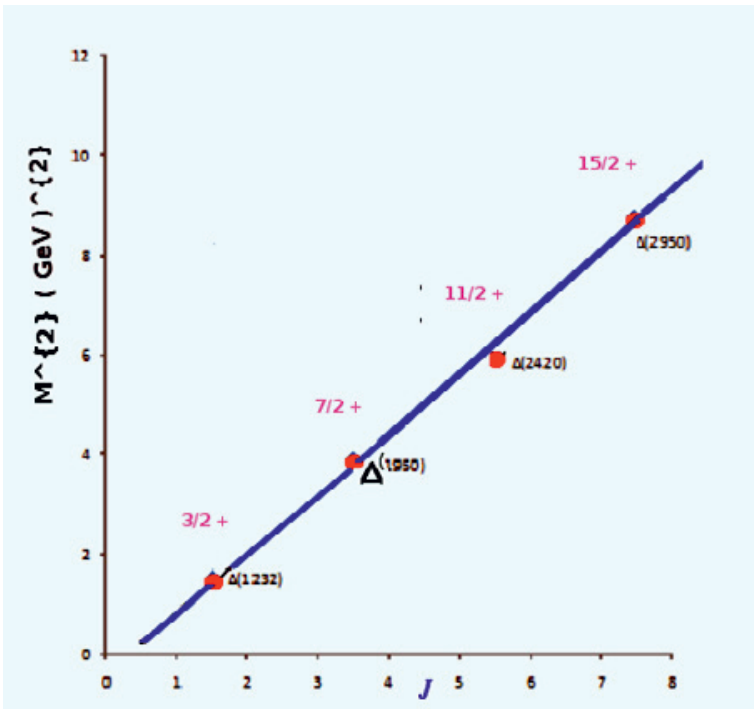

Fig. 4 : Regge trajectory of $\Delta$ baryons comprising $2 J^{P}=(3,7,11,15)^{+}$.

Eq. 17 reduces to

$$
\begin{aligned}
& \stackrel{\circ}{\mathcal{L}}_{N}=-\left(\sum_{\alpha=1}^{N}{\stackrel{\circ}{m_{\alpha}}}\right)\left[1-\frac{1}{K_{N}} v^{2}\right]^{\frac{1}{2}} ; v^{2}=\sum_{\beta=1}^{N} \vec{v}_{\beta} \vec{v}_{\beta} \\
& \longrightarrow \stackrel{\circ}{m}=\sum_{\alpha=1}^{N} \stackrel{\circ}{m}_{\alpha} ; \stackrel{\circ}{m}_{\alpha}=\stackrel{\circ}{m}_{\beta}=\stackrel{\circ}{m} / N \quad \forall \alpha, \beta=1, \cdots, N
\end{aligned}
$$

In eq. $22 v=\sqrt{v^{2}}$ defines a Euclidean distance in a space over real numbers $R^{\widehat{d}}$ of dimension $\widehat{d}=d_{\text {space }} \times N$, with $d_{\text {space }} \rightarrow 3$, yielding the associated scalar product

$$
\underline{w}=\left\{\vec{w}_{1}, \cdots, \vec{w}_{N}\right\} \longrightarrow\left(\underline{v}_{2} \mid \underline{v}_{1}\right)=\sum_{\beta=1}^{N} \vec{v}_{2 \beta} \vec{v}_{1 \beta}
$$

In $R^{\widehat{d}}$ the transformation to barycentric coordinates defined in eq. 8

$$
\begin{aligned}
& \underline{x}=\left\{\vec{x}_{1}, \cdots, \vec{x}_{N}\right\} \longrightarrow \underline{z}=\underline{z}(\underline{x})=\left\{\vec{z}_{1}, \cdots, \vec{z}_{N}\right\} \\
& \hline \vec{z}_{1}=\frac{1}{\sqrt{2}}\left(\vec{x}_{1}-\vec{x}_{2}\right) \\
& \vec{z}_{2}=\frac{1}{\sqrt{6}}\left(\vec{x}_{1}+\vec{x}_{2}-2 \vec{x}_{3}\right) \\
& \cdots \\
& \vec{z}_{v}=(v(v+1))^{-1 / 2}\left(\sum_{\alpha=1}^{v}\left(\vec{x}_{\alpha}-\vec{x}_{v+1}\right)\right) \\
& \cdots \\
& \vec{z}_{N-1}=\cdots ;(v=N-1) \\
& \vec{z}_{N}=N^{-1 / 2} \sum_{\alpha=1}^{N} \vec{x}_{\alpha}=N^{1 / 2} \vec{X}_{c . m .} .
\end{aligned}
$$




\section{ICNFP 2013}

is an orthogonal $O \widehat{d}$ mapping, i.e. preserves the scalar product defined in eq. 23. ${ }^{4}$ The reduction from the $O \widetilde{d}$ transformation, to be proven orthogonal, to its form in $R^{\widetilde{d}}=R^{N} \otimes R^{d_{\text {space }}}$

$$
\begin{aligned}
& O^{(\widehat{d})}=O^{(N)} \otimes \mathbb{I}_{d_{\text {space }} \times d_{\text {space }}} ; O^{(N)} \longleftrightarrow O_{\mu v} ; \\
& \mu, v=1, \cdots, N \text { with } \vec{z}_{\mu}=O_{\mu v} \vec{w}_{\nu}
\end{aligned}
$$

is not presented here. The proof of orthogonality of $O^{(\widehat{d})}$ reduces to establish orthogonality of the matrix $O^{(N)}$ displayed in eq. 25 .

Two relations follow independently from the c.m. constrant in configuration space $\vec{X}_{c . m}$. $\sim 0$ from eqs. $21-25$

$$
\begin{aligned}
\sum_{\alpha=1}^{N} \vec{x}_{\alpha}^{2} & =\quad \sum_{\beta=1}^{N-1} \vec{z}_{\beta}^{2}+N \vec{X}_{c . m .}^{2} \\
& =\sum_{\alpha=1}^{N}\left(\vec{x}_{\alpha}-\vec{X}_{c . m .}\right)^{2}+N \vec{X}_{c . m .}^{2} . \\
\longrightarrow \sum_{\beta=1}^{N-1} \vec{z}_{\beta}^{2} & =\sum_{\alpha=1}^{N}\left(\vec{x}_{\alpha}-\vec{X}_{c . m .}\right)^{2}
\end{aligned}
$$

\section{3 $\stackrel{\circ}{\mathcal{L}}_{N} \longrightarrow$ constructing canonical variables of Hamiltonin quantum mechanics}

$$
\begin{aligned}
& \vec{p}_{\beta}=\stackrel{\circ}{\mathcal{L}}_{N, \vec{v}_{\beta}}=\frac{\stackrel{\circ}{m}}{K_{N}}\left[1-\omega^{2}\right]^{-1 / 2} \frac{1}{2} v_{, \vec{v}_{\beta}}^{2} \\
& =\frac{\stackrel{\circ}{K_{N}}}{\beta}=1, \cdots . N \\
& \omega^{2}=\frac{1}{K_{N}} \sum_{\gamma=1}^{N} v_{\gamma}^{2} ; K_{N} \geq \frac{N}{N-1} ; \\
& \stackrel{\circ}{m}=\sum_{\alpha=1}^{N} \stackrel{\circ}{m}_{\alpha}
\end{aligned}
$$

Eq. 27 establishes the $\mathrm{N}$ canonically conjugate pairs of $d_{\text {space }}-$ vectors

$$
\left\{\vec{p}_{\beta}, \vec{x}_{\beta}\right\} ; \beta=1, \cdots, N
$$

generated through $\stackrel{\circ}{\mathcal{L}}_{N}$ with general properties outlined in eq. 22 . These variables do not satisfy the c.m. equivalence $\longrightarrow$ constraints defined in eqs. $10-12$ collected in eq. 29 below

$$
\begin{aligned}
& \vec{\pi}_{N}=N^{-1 / 2} \sum_{\alpha=1}^{N} \vec{p}_{\alpha} \rightarrow 0 \quad, \quad \vec{z}_{N}=N^{-1 / 2} \sum_{\alpha=1}^{N} \vec{x}_{\alpha} \rightarrow 0 \\
& \left(\vec{\pi}_{1}, \vec{z}_{1}\right), \cdots,\left(\vec{\pi}_{N-1}, \vec{z}_{N-1}\right) ; \vec{\pi}_{N}=0, \vec{z}_{N}=0 \\
& \vec{x}_{v} \underset{c . m .}{\sim} \vec{x}_{v}-\vec{X} ; v=1, \cdots, N ; \vec{X}=\frac{1}{N}\left(\sum_{\varrho=1}^{N} \vec{x}_{\varrho}\right)
\end{aligned}
$$

Omitting many details, we proceed to construct the main conserved quantity, following the original ref. 4 - [4]

$$
\begin{aligned}
& \mathcal{H}_{N}=\sum_{\alpha=1}^{N} \vec{v}_{\alpha} \vec{p}_{\alpha}-\stackrel{\circ}{\mathcal{L}}_{N} ; \sum_{\alpha=1}^{N} \vec{v}_{\alpha} \vec{p}_{\alpha}=\omega^{2}\left[1-\omega^{2}\right]^{-1 / 2} \\
& \stackrel{\circ}{\mathcal{L}}_{N}=-\stackrel{\circ}{m}\left[1-\omega^{2}\right]^{1 / 2} \longrightarrow \mathcal{H}_{N}=\stackrel{\circ}{m}\left[1-\omega^{2}\right]^{-1 / 2}
\end{aligned}
$$

\footnotetext{
4 in the approximately realistic case we have $N \equiv N_{c}=d_{\text {space }}=N_{f l}=3$ exactly.
} 
From eqs. 27 and 30 we infer

$$
\begin{aligned}
K_{N} \vec{p}_{\beta}^{2}=\mathcal{H}_{N}^{2} \frac{1}{K_{N}} \vec{v}_{\beta}^{2} \longrightarrow K_{N} \sum_{\gamma=1}^{N} \vec{p}_{\gamma}^{2} & =\mathcal{H}_{N}^{2} \omega^{2} \\
& =\mathcal{H}_{N}^{2}-\stackrel{\circ}{m}^{2}
\end{aligned}
$$

The last relation on the lower right side of eq. 31 follows from the decomposition

$$
\omega^{2}=1-\left(1-\omega^{2}\right)
$$

and eqs. 27 and 30. The structure of a genuine harmonic oscillator is brought a step nearer rearranging eq. 31

$$
K_{N} \sum_{\gamma=1}^{N} \vec{p}_{\gamma}^{2}+\stackrel{\circ}{m}^{2}=\mathcal{H}_{N}^{2}
$$

Since eq. 32 derives from a variational principle and yields consistent equations of motion both in the classical as well as the quantized interpretation, the Ansatz for $\stackrel{\circ}{m}^{2}$ realizing a system of $s=(N-1) d_{\text {space }}$ linear harmonic oscillators 5 implies the choice

$$
\begin{aligned}
& \stackrel{\circ}{m}^{2} \propto \sum_{\gamma=1}^{N}\left(\vec{x}_{\beta}-\vec{X}_{c . m .}\right)^{2}+\delta_{m^{2}} ; \\
& \delta_{m^{2}}: \text { configuration space independent }
\end{aligned}
$$

The constant transforming the proportionality in eq. 33 into an equality has to be determined in agreement with canonically conjugate momenta and positions .

The result is using also the relations in eq. 26

$$
\begin{aligned}
\mathcal{H}_{N}^{2} & =\left[\begin{array}{ll}
\left.\left.K_{N} \sum_{\alpha=1}^{N}\left(\vec{p}_{\alpha}\right)^{2}\right|_{\sum_{\beta=1}^{N} \vec{p}_{\beta}=0}+\stackrel{\circ}{m}^{2}\left(x_{\gamma}-X\right)\right] \\
= \\
+\left.\frac{\Lambda^{2}}{K_{N}} \sum_{\alpha=1}^{N}\left(\vec{x}_{\alpha}\right)^{2}\right|_{\sum_{\beta=1}^{N} \vec{x}_{\beta}=0}+\left.\right|_{\sum_{\beta=1}^{N} \vec{p}_{\beta}=0}+
\end{array}\right]
\end{aligned}
$$

Eq. 34 reveals that $\mathcal{H}_{N}^{2}$ so defined does satisfy the c.m.-constraints, and thus by Poincaré invariance is the mass-square operator, henceforth denoted $\mathcal{M}^{2} \equiv \stackrel{\circ}{\mathcal{M}}^{2}$. For simplicity of notation the superfix $\circ$, which stands for the chiral limit, in $\mathcal{M}^{2}$, is suppressed.

Next we transform momenta and positions to barycentric coordinates and cast the relations in eqs. 32 - 34 into the form

$$
\stackrel{\circ}{m}^{2}\left(x_{\gamma}-X\right)=\frac{\Lambda^{2}}{K_{N}} \sum_{\alpha=1}^{N-1} \vec{z}_{\alpha}^{2}+\delta_{m^{2}}
$$

In eq. $35 \Lambda$, of dimension mass-square, is the spring-tension ${ }^{6} . \Lambda^{2}$ is related to the gauge boson condensate of QCD ; a detailed discussion can be found in ref. $20-$ [20] .

\footnotetext{
5 For the realistic case $N=d_{\text {space }}=3, \mathrm{~s}$ is $=6$.

6 Spring is not string.
} 


\section{ICNFP 2013}

We arrive at the relation for $\mathcal{M}^{2}$ expressed through

$\widehat{d}_{c . m}=(N-1) d_{\text {space }}$ unconstrained, canonically conjugate pairs

$\left(\vec{\pi}_{1}, \vec{z}_{1}\right), \cdots,\left(\vec{\pi}_{N-1}, \vec{z}_{N-1}\right)$ defined in eq. 8

$$
\begin{aligned}
& \mathcal{M}^{2}=\left[K_{N} \sum_{\alpha=1}^{N-1}\left(\vec{\pi}_{\alpha}\right)^{2}+\frac{\Lambda^{2}}{K_{N}} \sum_{\alpha=1}^{N-1}\left(\vec{z}_{\alpha}\right)^{2}+\delta_{m^{2}}\right] \\
& \vec{\pi}_{\alpha}=\frac{1}{i} \partial_{\vec{z}_{\alpha}} ; \alpha=1, \cdots, N-1 ;\left[\vec{\pi}_{\beta k}, \vec{z}_{\gamma l}\right]=\frac{1}{i} \delta_{\beta \gamma} \delta_{k m} \mathbb{I} \\
& k, l=1, \cdots, d_{\text {space }}
\end{aligned}
$$

\section{The eigenvalues of $\mathcal{M}^{2}$ (eq. 36 )}

The eigenvalues - denoted $\mu^{2}$ - of $\mathcal{M}^{2}$ are obtained as solutions to the stationary differential equation for a wave function over unconstrained $\widehat{d}_{c . m}$. unconstrained barycentric position variables which shall be denoted $\underbrace{z}$

$$
\begin{aligned}
& \mathcal{M}^{2} \Psi(\underbrace{z})=\mu^{2} \Psi(\underbrace{z}) \\
& \underbrace{z}=\left\{z_{1}^{k}, \cdots z_{N-1}^{k}\right\} ; k=1, \cdots, d_{\text {space }}
\end{aligned}
$$

We introduce a parameter $\lambda$ of dimension mass in rescaling canonical variables

$$
\begin{aligned}
& \underbrace{\pi}=\lambda \underbrace{\Pi} ; \underbrace{z}=\lambda^{-1} \underbrace{\xi} \text { with } \underbrace{\Pi}, \underbrace{\xi} \text { dimensionless ; } \\
& \text { such that } \\
& \mathcal{M}^{2}= \\
& =\left(K_{N} \lambda^{2} \sum_{\alpha=1}^{N-1}\left(\vec{\Pi}_{\alpha}\right)^{2}+\frac{\Lambda^{2}}{K_{N} \lambda^{2}} \sum_{\alpha=1}^{N-1}\left(\vec{\xi}_{\alpha}\right)^{2}\right)+ \\
& +\delta_{m^{2}}
\end{aligned}
$$

and

$$
\begin{aligned}
& K_{N} \lambda^{2}=\frac{\Lambda^{2}}{K_{N} \lambda^{2}} \longrightarrow \lambda^{2}=\frac{\Lambda}{K_{N}} \\
& \mathcal{M}^{2}=\begin{array}{c}
\Lambda\left(K_{N} \lambda^{2} \sum_{\alpha=1}^{N-1}\left(\vec{\Pi}_{\alpha}\right)^{2}+\sum_{\alpha=1}^{N-1}\left(\vec{\xi}_{\alpha}\right)^{2}\right) \\
\quad+\delta_{m}{ }^{2}
\end{array}
\end{aligned}
$$

We transform eq. 38 into a countable $\widehat{d}_{c . m}$ - dimensional grid of dice-like boxes of unit length

$$
\begin{aligned}
& \stackrel{M}{M}^{2}=\alpha^{\prime} \mathcal{M}^{2}=\sum_{\{I\}} \frac{1}{2}\left[-\partial_{\xi_{I}}^{2}+\xi_{I}^{2}\right]+\alpha^{\prime} \delta_{m}{ }^{2} \\
& I(k, \alpha)=\alpha+(k-1)(N-1) ; k=1, \cdots, d_{\text {space }} \\
& 1 / \alpha^{\prime}=2 \Lambda \sim 1.06 \mathrm{GeV}^{2}
\end{aligned}
$$

In eq. $39\{I\}$ denotes the index set containing the integers

$$
\begin{aligned}
& I(k, \alpha)=\alpha+(k-1)(N-1) \\
& I=1, \cdots,(N-1) d_{\text {space }}=6 \text { for } N=3 ; d_{\text {space }}=3
\end{aligned}
$$


The absorption and creation operator are derived from the bosonic, dimensionless canonical pairs $\Pi_{I}=\left(\frac{1}{i} \partial_{\xi_{I}}, \xi_{I}\right) ;\left[\Pi_{J}, \xi_{I}\right]=\delta_{J I}$ II , for $J, I=1, \cdots, 6$ from here on specializing to the realistic values $N-3 ; d=3$.

$$
\begin{aligned}
& a_{I}=\frac{1}{\sqrt{2}}\left(\xi_{I}+i \Pi_{I}\right)=\frac{1}{\sqrt{2}}\left(\xi_{I}+\partial_{\xi_{I}}\right) \\
& a_{I}^{*}=\frac{1}{\sqrt{2}}\left(\xi_{I}-i \Pi_{I}\right)=\frac{1}{\sqrt{2}}\left(\xi_{I}-\partial_{\xi_{I}}\right)
\end{aligned}
$$

The main properties of the oscillators - in the linear oscillator mode basis - defined in eq. 41 are shown in the next equation, including eq. 41 for a concise overview

$$
\begin{aligned}
& a_{I}=\frac{1}{\sqrt{2}}\left(\xi_{I}+i \Pi_{I}\right)=\frac{1}{\sqrt{2}}\left(\xi_{I}+\partial_{\xi_{I}}\right) \\
& a_{I}^{*}=\frac{1}{\sqrt{2}}\left(\xi_{I}-i \Pi_{I}\right)=\frac{1}{\sqrt{2}}\left(\xi_{I}-\partial_{\xi_{I}}\right) \\
& \hline\left[a_{I}, a_{J}^{*}\right]=\delta_{I J} \text { II } ; J=1, \cdots, 6 ; \\
& \text { all other commutators }=0 \\
& \hline \text { self-adjoint counting operators }: \widehat{n}_{I}=a_{I}^{*} a_{I} ; \\
& \text { with eigenvalues } n_{I}=0,1, \cdots, \infty \\
& {\left[\widehat{n}_{I}, \widehat{n}_{J}\right]=0 \rightarrow\left\{\begin{array}{c}
\frac{\hat{n}}{n}=\left(\widehat{n}_{1}, \cdots, \widehat{n}_{6}\right) \\
\left.\underline{n}, \cdots, n_{6}\right)
\end{array}\right\}} \\
& \widehat{n}_{\bullet}=\frac{1}{2}\left[-\partial_{\xi}^{2}+\xi^{2}\right]+\text { zero point oscillations } \\
& \cdot: \text { generic for } I
\end{aligned}
$$

Substituting the last relation in eq. 42 into eq. 39 we obtain

$$
\begin{aligned}
\mathcal{M}^{2} & =\sum_{\{I\}} \widehat{n}_{I}+\left(\alpha^{\prime} \delta_{m^{2}}+3\right)=\Delta \mathcal{M}^{2}+C_{0} \\
\Delta \mathcal{M}^{2} & =\sum_{I=1}^{6} \widehat{n}_{I} ; C_{0}=\alpha^{\prime} \delta_{m^{2}}+3
\end{aligned}
$$

All quantities in eq. 43 - of natural dimension mass-square - are multiplied by $\alpha^{\prime} \sim 0.94 \mathrm{GeV}^{-1}$ and are ( or rendered) dimensionless .

Eq. 37 becomes

$$
\begin{aligned}
& \Delta \mathcal{M}^{2} \Psi(\underbrace{z})=\mathbb{N} \Psi(\underbrace{z}) ; \mathcal{M}^{2}=\Delta \mathcal{M}^{2}+C_{0} \\
& \underbrace{\left.z_{1}^{k}, \cdots z_{N-1}^{k}\right\} ; k=1, \cdots, 3}_{\mathbb{N}=\sum_{I=1}^{6} n_{I} \longleftrightarrow \text { compare in contrast with eq. } 5}
\end{aligned}
$$

In eq. $44 C_{0}$ is to be considered a phenomenological parameter - not ( naturally ) an integer determining e.g. the lowest mass-square of a Regge trajectory, identified approximately to be linear and part of the ( an ) $\mathbb{N}$ - multiplet of baryons .

Finding all finite $\mathbb{N}$ solutions to the first equation in eq. 44 and their multiplicities for fixed $\mathbb{N}$ - compatible with fermion statistics for quark fields and baryons - defines the 'Counting' in the title of the present discussion. 


\section{Concluding remarks, outlook}

1) A different view on resonances from QCD sum rules and condensates, also relating to supersymmetric QCD by Adi Armoni and Mikhail Shifman can be found in ref. 21 - [21].

2) The $(70 \times \vec{L}=1)^{-}$

negative parity u, $\mathrm{d}, \mathrm{s}$ baryon multiplet with $\mathbb{N}=1$ is well described in the current PDG review [7] - 'Quark Model' by C. Amsler, T. De Grand and B. Krusche in ref. 22 - [22]. Extended other baryon- and meson multiplets including heavy quark flavors $\mathrm{c}, \mathrm{b}$ are assigned quark and antiquark configurations as well . A pioneering paper discussing $\mathrm{u}, \mathrm{d}, \mathrm{s}$ flavored $\bar{q}^{\prime} q$ mesons is due to George Zweig in ref. $23-[23]$.

3) The circular pair-mode oscillator basis

is presented in detail in ref. $1-[1]$. The scope of this outline unfortunately does not allow to cover this topic, instrumental to establish the counting of oscillatory modes in $\mathrm{u}$. $\mathrm{d}, \mathrm{s}-$ baryons .

4) The $\mathbb{N}$ - density of baryon states per mass-square

defined in eq. 1

$$
\varrho_{n}\left(m^{2}\right)=\frac{\partial \mathbb{N}\left(m^{2}\right)}{\partial m^{2}}
$$

behaves for large $\mathbb{N}$ like $\mathbb{N}^{u} ; u=5$. This is enough to establish that string modes and oscillator modes presented are inequivalent.

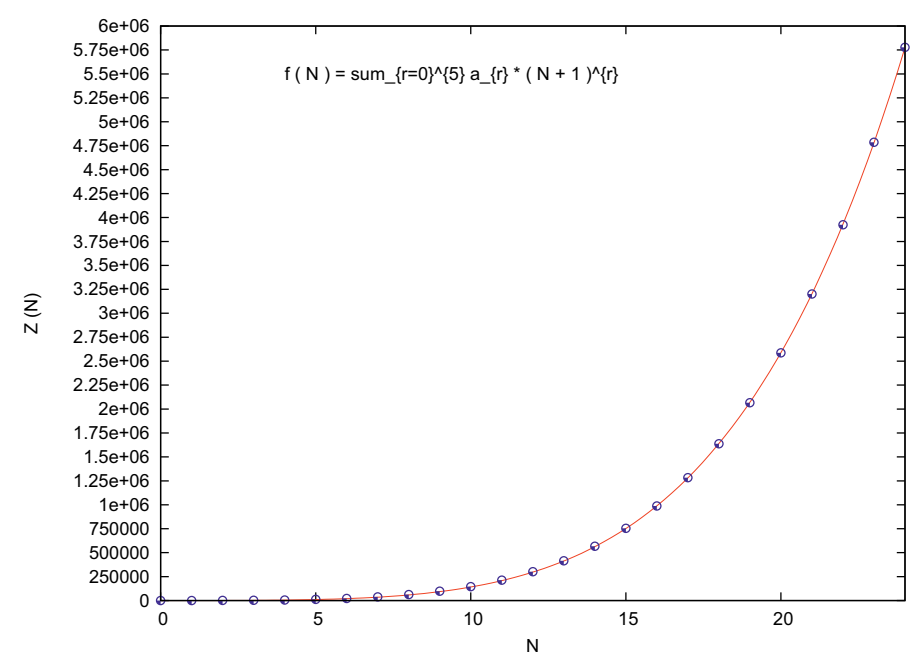

Fig. $5: \#(\mathbb{N})$

4) (continued) missing states

Counting all $\mathrm{u}, \mathrm{d}, \mathrm{s}$ baryon states in the PDG - [7] with angular momentum and isospin multiplicities accounted for, 1274 are obtained, omitting a few very doubtful resonances . 
The count of states with $\mathbb{N} \leq \mathbb{N}^{*}$ gives

\begin{tabular}{|rr|}
\hline $\mathbb{N}^{*}$ & $\#$ \\
\hline 0 & 56 \\
1 & 266 \\
2 & 1310 \\
3 & 4090 \\
\hline
\end{tabular}

with $\mathbb{N}^{*} \sim 3$ beeing a fair estimate of resonances up to $2.5-3 \mathrm{GeV}$.

5) outlook

We hope that at the high energy frontier, despite the odds looking unfavorable, exploiting the increased production cross sections of hitherto unobserved resonances, the art of resonance spectroscopy - even of low energy resonances - can witness a new frontier .

\section{References}

[1] P. Minkowski , 'Oscillatory modes of quarks in baryons for 3 quark flavors u , d , s', URL : http://www.mink.itp.unibe.ch in 'Lectures and talks', file : oscimodes-fl6.pdf.

[2] P. Minkowski, 'Count of oscillatory modes of quarks in baryons for 3 quark flavors u, d, s', internal report .

[3] S. Kabana and P. Minkowski, 'On the thermal phase structure of QCD at vanishing chemical potentials', CERN-PH-TH-2010-002, Jan 2010. 7pp., Int.J.Mod.Phys. A26 (2011) 3035-3050, arXiv:1001.0707 [hep-ph], and references cited therein .

[4] P. Minkowski, 'On the oscillatory modes of quarks in baryons', March 1980, Nucl. Phys. B174 (1980) 258-268 and references cited therein .

[5] G. F. Chew , 'S-matrix theory of strong interactions without elementary particles', Rev. Mod. Phys. 34 (1962) 394 ;

S. Frautschi, M. Gell-Mann and F. Zachariasen, 'Experimental consequences of the hypothesis of Regge poles', Phys. Rev. 126 (1962) 2204.

[6] P. D. B. Collins and E. J. Squires, 'Regge Poles in Particle Physics', Springer Tracts in Modern Physics 45 , G. Höhler ed., Springer-Verlag Berlin Heidelberg New York 1968 .

[7] J. Beringer et al. (Particle Data Group), Phys. Rev. D86 (2012) 010001.

[8] R. Hagedorn, 'Statistical thermodynamics of strong interactions at high energy', Nuovo Cimento Suppl. 3 (1965) 147-186.

[9] G. Veneziano, 'Construction of a crossing - symmetric, Regge behaved amplitude for linearly rising trajectories', Nuovo Cim. A57 (1968) 190-197.

[10] J. Schwarz, 'Superstring Theory', Physics Reports 89 (1982) 223.

[11] Enrique Alvarez (CERN), M.A.R. Osorio (Madrid, Autonoma U.), 'Superstrings at Finite Temperature', Oct 1986, 22 pp., CERN-TH-4571/86, Phys.Rev. D36 (1987) 1175 .

[12] Steven S. Gubser (Princeton U.), Sergei Gukov (Princeton U. \& Caltech \& CIT-USC), Igor R. Klebanov (Princeton U.), Mukund Rangamani (Princeton U. \& Caltech \& CIT-USC), Edward Witten (Caltech \& CIT-USC \& Princeton, Inst. Advanced Study), 'The Hagedorn transition in non-commutative open string theory', Sep 2000. 22 pp., PUPT-1949, CALT-68-2296, CITUSC00-050, J.Math.Phys. 42 (2001) 2749-2764 .

[13] 'Handbook of mathematical functions', M. Abramowitz and I. A. Stegun eds., Dover Publications, Inc., New York 1970 .

[14] Michael B. Green, John H. Schwarz, Edward Witten, 'Superstring Theory', 2 Volume Set 25th Anniversary Edition ,- Cambridge Monographs on Mathematical Physics 2012. 
[15] J. Zinn-Justin, 'Quantum field theory and critical phenomena', Oxford University Press, Oxford 2002 , and references cited therein .

[16] P. Minkowski, 'Light quark mass ratios (mu:md:ms) from meson and bayyon mass splittings', Modern Physics Letters A Vol. 28, No. 25 (2013) 1360015 and references cited therein .

[17] J. H. Kühn, 'Precise heavy quark masses', Modern Physics Letters A 28, No. 25 (2013) 1360019.

[18] C. L. Critchfield, 'Scalar binding of quarks', Phys. Rev. D. 12 (1975) 923-925, and C. L. Critchfield, 'Scalar potentials in the Dirac equation', J. Math. Phys. 17 (1976) 261 ; http://dx.doi.org/10.1063/1.522891 (6 pages) .

[19] Shuchi Bisht, Navjot Hothi† and Gaurav Bhakuni, 'Phenomenological Analysis of Hadronic Regge Trajectories', EJTP 7, No. 24 (2010) 299-318.

[20] P. Minkowski, 'Embedding oscillatory modes of quarks in baryons in QCD', URL : http://www.ccsem.infn.it/issp2013/index.html, and references cited therein.

[21] A. Armoni (CERN), M. Shifman (Minnesota U.), 'Strings in the Yang-Mills theory: How they form, live and decay', Sep 2003. 16 pp., published in Springer Proc.Phys. 98 (2005) 415-430, prepared for Conference: C03-09-04.1, p.415-430 Proceedings ,

Bibtex : @articleTrampetic:2005ib, author = "Trampetic, J. and Wess, J.", editor = "Trampetic, J.", title = "Particle physics and the universe. Proceedings, 9th Adriatic Meeting, Dubrovnik, Croatia,September 4-14, 2003", year = "2005",SLACcitation $=$ "\% \%CITATION = INSPIRE692435;\%\%".

[22] 'The Review of Particle Physics', PDG, J. Beringer et al, Review : 14. QUARKMODEL, Revised August 2011 by C. Amsler (University of Zurich), T. DeGrand (University of Colorado, Boulder) and B. Krusche (University of Basel) .

[23] G. Zweig , "Meson classification in the quark model", in "Meson Spectroscopy", edited by C. Baltay and A. H. Rosenfeld, 1968 , 485-496. 